\title{
EVALUATION OF SOME PARAMETERS OF COPPER METABOLISM AND LEIPZIG SCORING SYSTEM IN THE DIAGNOSIS OF WILSON DISEASE
}

\author{
Diana Gancheva ${ }^{1}$, Iskren Kotzev ${ }^{1}$, Mariana Kaneva ${ }^{2}$ \\ ${ }^{1}$ Clinic of Gastroenterology, St. Marina University Hospital of Varna, Medical University \\ of Varna, ${ }^{2}$ Department of Statistics, University of Economics of Varna
}

\begin{abstract}
PURPOSE: Wilson disease (WD) is an autosomal recessively inherited disorder of copper accumulation and toxicity. Its recognition is easy in the presence of typical clinical presentations. Unexplained liver test abnormalities are a diagnostic challenge and require more examinations. The objective of this study is to assess the diagnostic value of ceruloplasmin, 24-hour urine copper excretion and Leipzig scoring system in WD.

MATERIAL AND METHODS: Sixty-five patients with WD (22 females and 43 males) and a control group of 17 patients with other chronic liver diseases (CLD) were analyzed. The values of the parameters of copper metabolism and Leipzig scoring system were evaluated.

RESULTS: Average ceruloplasmin level was under $0,2 \mathrm{~g} / \mathrm{L}$ and 24-hour urinary copper concentration was increased. D-penicillamine challenge test showed a mean value of $17,4 \mu \mathrm{mol} / 24$ hours of urinary copper excretion in WD patients versus $5,46 \mu \mathrm{mol} / 24$ hours in CLD ones. According to the Leipzig scoring diagnostic criteria, 58 WD patients $(89,23 \%$ of the cases) presented with a score $\geq 4$ (maximal value of 12 ). Score 3 was found out in seven patients, however, the exclusion of other etiology and the clinical course of the disease confirmed the diagnosis. The control subjects presented with a score $\leq 3$ as it was $\leq 2$ in $76,5 \%$ of the cases.

CONCLUSION: Our results confirm the diagnostic value for WD of the Leipzig scoring system combined with clinical symptoms, laboratory parameters of copper metabolism, genetic testing and liver biopsy in clinical practice.
\end{abstract}

Keywords: Wilson disease, Leipzig scoring system, ceruloplasmin, urine copper excretion, D-penicillamine test

Address for correspondence

Diana Gancheva, MD

Clinic of Gastroenterology

St. Marina University Hospital of Varna

1 Hristo Smirnenski Str.

9010 Varna, Bulgaria

e-mail:gancheva_vn@abv.bg

Received: February 7, 2014

Accepted: February 11, 2014

\section{INTRODUCTION}

Wilson's disease (WD) is a rare autosomal hereditary recessive disorder of copper metabolism. It is characterized by progressive copper accumulation and toxic action in the organism, mainly in the liver, brain, cornea, and kidneys. The disease can be treated in the case of timely recognition $(3,6)$. The diagnosis is easy in the presence of typical manifestations. A vague liver disease or isolated symptoms originating 
from other organs and systems present a diagnostic challenge and require additional examinations $(8,16)$.

The objective of this study is to reveal the diagnostic value of serum ceruloplasmin, 24-hour urinary copper excretion and Leipzig scoring system in WD.

\section{MATERIAL AND METHODS}

The study covered $65 \mathrm{WD}$ patients, 22 females and 43 males at a mean age of $37,75 \pm 12,98$ years (range, 18-65 years) hospitalised and followed-up in the Clinic of Gastroenterology, St. Marina University Hospital of Varna, during the period from 2003 till 2013 as well as 17 patients with other chronic liver diseases (CLD) serving as controls. The levels of serum ceruloplasmin as well as of basal and provoked 24-hour urinary copper expression were examined. Leipzig scoring system for WD diagnosis was used for the evaluation of the patients from both groups.

This system includes both clinical and laboratory parameters with score range between one and four. The diagnosis of WD is established in case of $\geq 4$ scores. In case of 3 scores, the diagnosis is possible and additional examinations are needed while in case of $\leq 2$ scores, the diagnosis is less probable.

The following statistical methods were made use of: descriptive statistics, analysis of univariate and multivariate empirical distributions, independent samples test, and t-test for equality of means. A p-value of 0,05 was defined as statistically significant. Statistical data processing was done by using of SPSS 13.0 software for Windows and STATISTICA 5, StatSoft Inc., for Windows and MS Excel 2002.

\section{RESULTS AND DISCUSSION}

Both serum ceruloplasmin and urinary copper excretion represent the basic parameters of copper metabolism assessment.

\section{Ceruloplasmin}

Ceruloplasmin is a copper-bearing protein that binds approximately $90 \%$ of circulating copper in healthy individuals. Its normal concentration is $0,2-$ $0,4 \mathrm{~g} / \mathrm{L}$ and values less than $0,2 \mathrm{~g} / \mathrm{L}$ suggest the presence of WD. The estimation of the serum ceruloplasmin is a useful diagnostic test. This level is, usually, abnormally low in WD. However, it should be kept in mind that $10-20 \%$ of the patients present with cerulo- plasmin level at the low borderline or within normal limits (1,2,15-17). Ceruloplasmin is an acute-phase protein and its concentration increases in response to inflammation, infection and injury and that is why falsely-elevated as well as normal values can occur in some patients. On the other hand, diminished concentrations can be established under conditions of malnutrition and hypoproteinemia.

Our own data indicate that serum ceruloplasmin values are lower in WD patients than in those with other CLD (Fig. 1).

In $55 \mathrm{WD}$ patients $(84,62 \%$ of the cases), the value of this parameter remains below the reference limits while in 10 ones $(15,38 \%$ of the cases) it is within the normal range that is close to the results reported by other authors $(8,11)$.

Comparison of ceruloplasmin levels between the patients with WD and with other CLD

Statistical hypothesis testing concerning the mean ceruloplasmin values and according to the logic of t-test in independent samples has been carried out. These values are statistically significantly lower in the patients with WD than in those with other $\mathrm{CLD}$, i. e. $0,149 \mathrm{~g} / \mathrm{L} \pm 0,067 \mathrm{~g} / \mathrm{L}$ versus $0,224 \mathrm{~g} / \mathrm{L} \pm 0,062$ $\mathrm{g} / \mathrm{L}$. Our data are similar to the results reported elsewhere and indicate the usefulness of this diagnostic parameter $(7,8,11)$. However, the cases with a normal serum ceruloplasmin level in our continent of WD patients should not be ignored and the diagnosis of the disease could not be excluded. The results from $\mathrm{t}$ test analysis are presented on Fig. 2.

\section{Urinary copper excretion}

Although urinary copper excretion is elevated in most WD patients it does not result in any negative copper balance (10). The measurement of 24-hour cupriuria is another reliable test. It is useful for the diagnosis of the disease and monitoring of the treatment as well. This parameter reflects the amount of copper that remains not bound to blood ceruloplasmin. A great part of copper is bound to albumins and amino acids. The copper that is bound to the amino acids is filtrated through the glomerules while albumin-bound copper is not filtrated except for the cases of significant proteinuria. Under these conditions, protein loss enhances cupriuria. Serum albumin is another factor that is probably involved in 


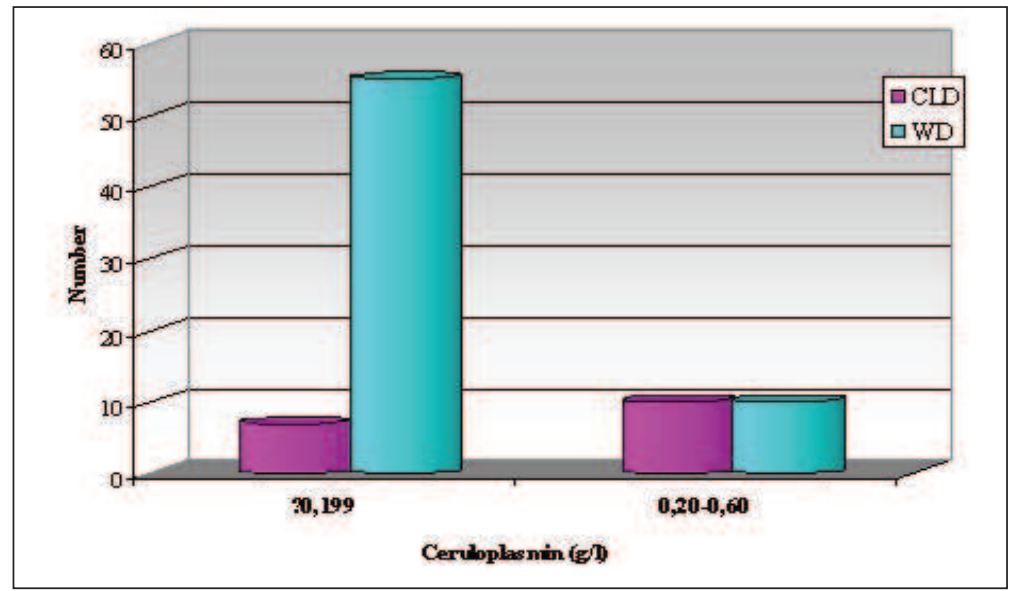

Fig. 1. Patient's distribution according to ceruloplasmin levels (in $g / L$ )

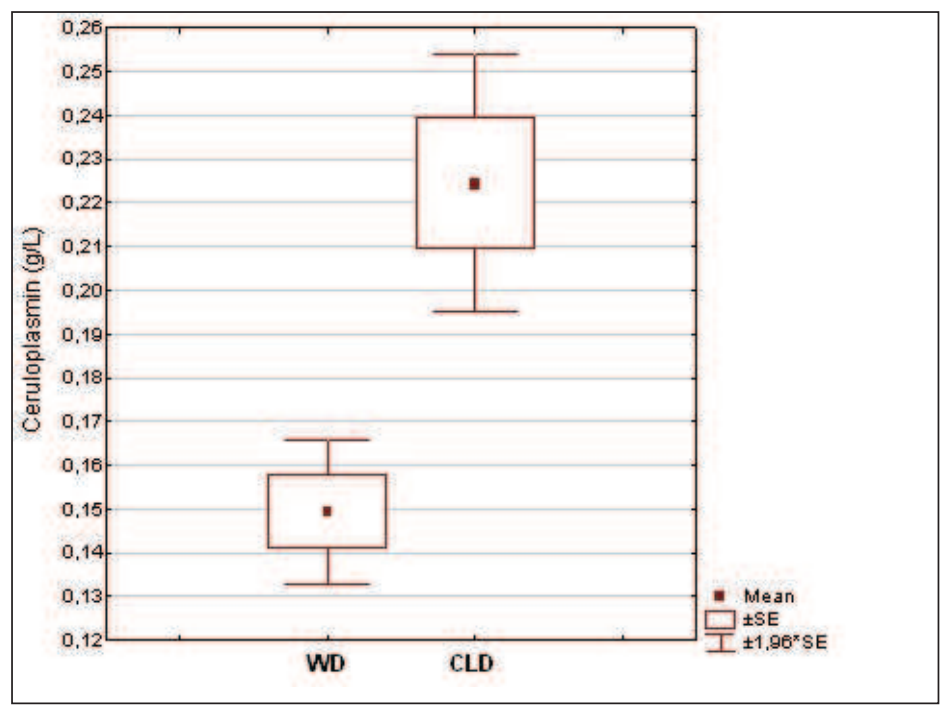

Fig. 2. Serum ceruloplasmin levels in patients with WD and with CLD

the determination of the copper amount excreted by the urine. The low albumin level will enable a greater quantity of copper to find weaker ligands thus enabling the more intensive copper filtration through the glomerules (19). It is accepted that cupriuria over $1,6 \mu \mathrm{mol} / 24$ hours i.e. over $100 \mu \mathrm{g} / 24$ hours is a reliable diagnostic test in a clinically manifested disease $(1,2,11,13-15,17)$. Some authors report a high specificity of this diagnostic test (11).The values over 0,64 $\mu \mathrm{mol} / 24$ hours are strongly indicative of WD and require further examinations $(3,16)$.
Comparative analysis of mean basal urinary copper values in WD and other CLD

The descriptive analysis demonstrates that the mean spontaneous level of cupriuria in WD patients is $4,67 \mu \mathrm{mol} / 24$ hours at a standard deviation of $4,666 \mu \mathrm{mol} / 24$ hours. These values are much higher than those in CLD patients. The considerable difference between the corresponding values can be seen on Table 1 and Table 2.

There is evidence that the mean values of spontaneous cupriuria between both groups of patients 
are statistically different. The equal variance assumed can reach up to $6,033 \mu \mathrm{mol} / 24$ hours while the equal variances not assumed can reach up to 4,973 $\mu \mathrm{mol} / 24$ hours at a confidence interval (CI) of $95 \%$ when these two groups are compared. However, this test can't be performed in patients with renal failure. Its interpretation is hampered by the presence of similar findings in other liver diseases such as autoimmune hepatitis, chronic cholestasis and acute liver failure. The considerable variations in WD patients are, probably, related to group heterogeneity including different age, time lag between the onset of symptoms and the diagnosis of WD, clinical form and severity of WD as well. The mean cupriuria value is by four times higher than the upper normal range and is considerably higher than that of the control group. That is why this parameter contributes to the correct diagnosis of WD.

Comparative analysis of mean provoked urinary copper concentrations in WD and other CLD

D-penicillamine-induced cupriuria, i. e. D-penicillamine challenge test, is an important and useful additional diagnostic test $(5,7,9,12,13,18)$. The level of induced 24-hour cupriuria is by five times higher the upper normal range in WD patients. It amounts to $17,403,13 \mu \mathrm{mol} / 24$ hours while the corresponding values in the patients with other CLD are by approximately three times lower $(5,46 \pm 2,99 \mu \mathrm{mol} / 24$ hours $)$. This difference is statistically significant and can be explained by a certain disorder of copper metabolism in WD (Table 3). This deals with the upper reference limits and the control group as well.

There is a five-fold increase over the normal range in $38 \mathrm{WD}$ patients as well as a ten-fold one in 10 of these patients. The elevation of copper excretion in comparison with its initial level by more than five times in $24 \mathrm{WD}$ patients is of importance, too. This fact enables us to consider this test useful, necessary and applicable diagnostic parameter, especially in the cases of inexplicable hepatomegaly, vague liver disease, elements of hemolysis or unspecified neurological disease.

\section{Leipzig scoring system for diagnosis of WD}

During the 8th International Conference on WD and Menkes disease in Leipzig in 2001, a scoring system for the diagnosis of WD was suggested. Its purpose is to warrant objective criteria of high specificity and sensitivity for WD. A combination of clini$\mathrm{cal}$ and laboratory tests within a score range between zero and four has been elaborated. It includes the following parameters: Kayser-Fleischer ring, neurological symptoms, serum ceruloplasmin, Coombs-negative hemolytic anemia, copper concentration in the hepatic tissue or staining for copper with rhodanine in the hepatocytes, spontaneous and/or provoked urinary copper excretion, and mutation analysis. This scoring system is incorporated into the clinical practice guidelines for WD approved by the European Association for Study of the Liver (EASL) in 2012 $(3,4)$.

In 2010, we introduced this system in the Clinic of Gastroenterology and subsequently evaluated all the patients with WD according to it. Along the parameters of copper metabolism, the rest items of this integrated scoring system were analyzed.

We established neurological symptoms in 35 patients. Out of them, 32 patients presented with a mixed hepatic and neurological form and the rest three did with solely neurological manifestations. Coombs-negative hemolytic anemia was proved in seven patients. Kayser-Fleischer ring was observed in 23 patients. DNA analysis was accomplished in 54 patients. In 26 of them (48,15\% of the cases), there were ATP7B gene mutations. Twenty-three patients $(35,38 \%$ of the cases) underwent percutaneous aspiration liver biopsy. A histochemical examination with rhodanine for copper detection was carried out in 19 patients. A positive result was obtained in 10 patients (52,63\% of the cases).

The distribution of the patients with WD and with other CLD according to the criteria of Leipzig scoring system is demonstrated on Fig. 3.

According to this system, 58 patients $(89,23 \%$ of the cases) present with a score sum over four that proves the diagnosis of WD. Score of four occupies the greatest relative share of WD patients - 26,2\%. The relative share of the patients with score of five and six is $12,3 \%$. The relative share of $7,7 \%$ is equal for the patients with scores of 7, 8 and 11 as well. Seven patients $(10,77 \%)$ present with score of three. In them, the viral, autoimmune and toxic reasons for a CLD have been excluded. Clinical observation and 
Table 1. Basal urinary copper concentrations in patients with WD and with CLD (descriptive statistics)

\begin{tabular}{lllll} 
Disease & $\mathbf{n}$ & $\begin{array}{l}\text { mean }(\mu \mathrm{mol} / 24 \\
\text { hours })\end{array}$ & $\begin{array}{l}\text { stand. dev. } \\
(\boldsymbol{\mu m o l} / \mathbf{2 4} \text { hours })\end{array}$ & $\begin{array}{l}\text { S.E. mean }(\boldsymbol{\mu m o l} / \mathbf{2 4} \\
\text { hours })\end{array}$ \\
WD & 61 & 4,670 & 4,666 & 0,597 \\
CLD & 17 & 0,871 & 0,362 & 0,088 \\
\hline
\end{tabular}

Table 2. Basal urinary copper concentrations in patients with WD and with CLD (results from a statistical hypothesis)

\begin{tabular}{|c|c|c|c|c|c|c|c|c|c|}
\hline \multirow[t]{2}{*}{ Assumptions } & \multicolumn{2}{|c|}{$\begin{array}{l}\text { Levene's test } \\
\text { for equality of } \\
\text { variances }\end{array}$} & \multicolumn{7}{|c|}{ t-test for equality of means } \\
\hline & $\mathrm{F}$ & Sign. & $\mathrm{t}$ & $\mathrm{df}$ & $\begin{array}{l}\text { Sign. } \\
\text { (2-tailed) }\end{array}$ & $\begin{array}{l}\text { Mean } \\
\text { diff. }\end{array}$ & S.E. diff. & $\begin{array}{l}95 \% \mathrm{C} \\
\text { lower }\end{array}$ & $\begin{array}{l}\text { difference } \\
\text { upper }\end{array}$ \\
\hline $\begin{array}{l}\text { equal } \\
\text { variances } \\
\text { assumed }\end{array}$ & 13,705 & 0,000 & 3,309 & 76 & 0,001 & 11,942 & 1,138 & 1,500 & 6,033 \\
\hline $\begin{array}{l}\text { equal } \\
\text { variances not } \\
\text { assumed }\end{array}$ & & & 6,237 & 62,516 & 0,000 & 11,942 & 0,604 & 2,559 & 4,973 \\
\hline
\end{tabular}

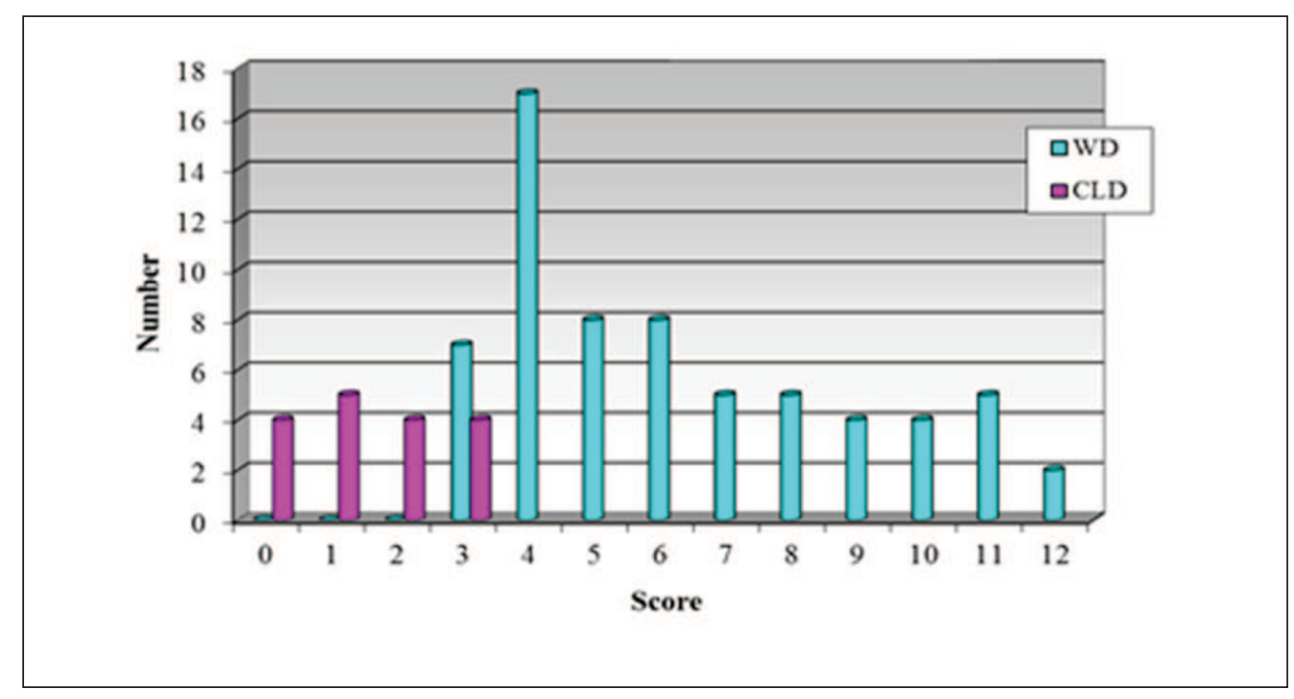

Fig. 3. Patients' distribution according to the Leipzig scoring system 
favourable therapeutic influence on the laboratory parameters has confirmed the diagnosis in these patients. Only four patients with other CLD present with score of three while the rest 13 ones $(76,47 \%)$ do with a score that is less or equal to two. The patients with a score of one occupy the greatest relative share in the control group. These results demonstrate that the parameters incorporated in the Leipzig scoring system are a reliable combination of criteria for the precise and definite diagnosis of WD. It is noteworthy that there does not exist a unique parameter for this diagnosis at all. On the other hand, it is not correct to accept that all the parameters should obligatorily be abnormal. In this respect, the complex evaluation, clinical observation and, in some cases, the therapeutic test with D-penicillamine could help the correct diagnosis.

\section{CONCLUSION}

The diagnosis of WD is based on the complex assessment of a combination of various clinical signs and laboratory parameters. Our results show that serum ceruloplasmin can be used mainly in combination with the rest of the parameters. We have proved the usefulness and necessity of the test of basal and, particularly, of the provoked urinary copper excretion for the correct diagnosis of WD. Our experience gained with the application of the Leipzig scoring system indicates that this is a reliable combination of clinical, laboratory, genetic and morphological parameters. It can be helpful for the precise diagnosis of $\mathrm{WD}$, especially in the patients with a subclinical liver damage with a view to the timely treatment and prevention of the complications of this potentially curable hereditary disease.

\section{REFERENCES}

1. Brewer, G. J. Recognition, diagnosis, and management of Wilson's disease.- Proc. Soc. Exp. Biol. Med. 2000; 223(1): 39-46.

2. Das, S. K., K. Ray. Wilson's disease: an update.Nat. Clin. Pract. Neurol. 2006; 2(9): 482-493.

3. EASL Clinical Practice Guidelines:Wilson's disease.- J. Hepatol. 2012; 56(3): 671-685.

4. Ferenci, P., K. Caca, G. Loudianos, G. MieliVergani, S. Tanner, I. Sternlieb, et al. Diagnosis and phenotypic classification of Wilson disease.Liver Int. 2003;23(3): 139-142.

5. Foruny, J. R., D. Boixeda, A. L. Sanroman, E. V. Sequeros, M. Villafruela, M. V. Romero, et al. Usefulness of penicillamine-stimulated urinary copper excretion in the diagnosis of adult Wilson's disease.- Scand. J. Gastroenterol. 2008;43(5): 597-603.

6. Kolarski, V. Wilson's disease.- In: Hepatology. Sofia, Tilia. 1998; 441-454 (in Bulgarian).

7. Mahjob, F., R. Fereiduni, I. Jahanzad, F. Farahmand, M. Monajemzadeh, M. Najafi. Atomic absorption spectrometry in Wilson's disease and its comparison with other laboratory tests and paraclinical findings.- Iran. J. Pediatr. 2012;22(1): 52-56.

8. Mansoor, S., A. K. Naveed, A. Majeed. Analysis of clinical and biochemical spectrum of Wilson disease patients.- Indian J. Pathol. Microbiol., 2012; 55(3): 365-369.

9. Martins da Costa, C., D. Baldwin, B. Portmann, Y. Lolin, A.P. Mowat, G. Mieli- Vergani. Value of urinary copper excretion after penicillamine challenge in the diagnosis of Wilson's disease. Hepatology. 1992;15(4); 609-615.

10. Mateva, L. Wilson's disease.- In: Internal medicine. Ed. by Z. Krastev. Sofia. 2004;272-273 (in Bulgarian).

11. Merle, U., M. Schaefer, P. Ferenci, W. Stremmel. Clinical presentation, diagnosis and long-term outcome of Wilson's disease: a cohort study. Gut. 2007;56(1): 115-120.

12. Moores, A., S. Fox, A. Lang, G. M. Hirschfield. Wilson disease: Canadian perspectives on presentation and outcomes from adult ambulatory setting. Can. J. Gastroenterol. 2012;26(6): 333-339.

13. Muller, T., S. Koppikar, R. Taylor, F. Carragher, B. Schlenck, P. Hainz-Erian, et al. Re-evaluation of the penicillamine challenge test in the diagnosis 
of Wilson's disease in children. J. Hepatol. 2007;47(2): 270-276.

14. Nicastro, E., G. Ranucci, P. Vajro, A. Vegnente, R. Iorio. Re-evaluation of diagnostic criteria for Wilson disease in children with mild liver disease. Hepatology. 2010;52(2): 1948-1956.

15. Roberts, E. A., M. L. Schilsky. Diagnosis and treatment of Wilson disease: An update. Hepatology. 2008;47(6): 2089-2111.

16. Rozencrantz, R., M. L. Schilsky. Wilson disease. Pathogenesis and clinical considerations in diagnosis and treatment. Semin. Liver Dis. 2011;31(3): 245-259.

17. Shah, R., M. Piper. Wilson disease. eMedicine Specialties, Gastroenterology, Systemic Disease. Updated, August 25, 2009.

18. Walshe, J. M. The pattern of urinary copper excretion and its response to treatment in patients with Wilson's disease. Q. J. Med. 2011;104(9): 775-778.

19. Walshe, J. M. Serum 'free' copper in Wilson disease. Q. J. Med. 2012;105(5): 419-423. 